\title{
1 Genome sequence and analysis of the eggplant (Solanum melongena $\mathbf{L}$.
}

3 Dandan $\mathrm{Li}^{1}$, Jun Qian ${ }^{2}$, Wenjia $\mathrm{Li}^{1}$, Yaqin Jiang ${ }^{1}$, Guiyun $\mathrm{Gan}^{1}$, Weiliu $\mathrm{Li}^{1}$, Riyuan $\mathrm{Chen}^{1}, \mathrm{Ning} \mathrm{Yu}^{1}$,

4 Yan $\mathrm{Li}^{1}$, Yongguan $\mathrm{Wu}^{1}$, Dexian Kang ${ }^{1}, \mathrm{Jinmin}_{\mathrm{Lian}}{ }^{2}$, Yongchao $\mathrm{Niu}^{2}$ and Yikui Wang ${ }^{1, *}$

5

$6 \quad{ }^{1}$ Institute of Vegetable Research, Guangxi Academy of Agricultural Sciences, Nanning, China

$7 \quad{ }^{2}$ Biozeron Shenzhen, Inc., Shenzhen, China

8 *Correspondence ( $\mathrm{Tel}+86-771-3186-372$; email ykwang@gxaas.net)

9 Running Head: Eggplant genome 


\section{Summary}

13 The eggplant (Solanum melongena L.) is one of the most important Solanaceae crops, ranking third in

14 the total production and economic value in the genus Solanum. Here, we report a high-quality,

15 chromosome-scale eggplant reference genome sequence of 1,155.8 Mb, with $\mathrm{N} 50$ of $93.9 \mathrm{Mb}$, which

16 was assembled by combining PacBio long reads and Hi-C sequencing data. Repetitive sequences

17 occupied $70.1 \%$ of the assembly length, and 35,018 high-confidence protein-coding genes were

18 annotated based on multiple evidence. Comparative analysis revealed 646 species-specific families

19 and 364 positive selection genes, conferring distinguishing traits to the eggplant. We performed

20 genome-wide identification of disease resistance genes and discovered an expanded gene family of

21 bacterial spot resistance in the eggplant and pepper but not in tomato and potato. The genes involved

22 in chlorogenic acid synthesis were comprehensively characterized. Highly similar chromosomal

23 distribution patterns of polyphenol oxidase genes were observed in the eggplant, tomato, and potato

24 genomes. The eggplant reference genome sequence will not only facilitate evolutionary studies in the

25 Solanaceae but also facilitate their breeding and improvement.

27 Keywords: eggplant, Solanum melongena, genome sequencing, evolution, disease resistance,

28 chlorogenic acid, transcription factors

\section{Introduction}

31 Solanaceae plants are medium-sized angiosperms; they are the largest group of vegetable crops and

32 the third largest group of economic plants. The taxa in the Solanaceae family are abundant and

33 diverse, with 90 genera and 3,000-4,000 species. This family includes many important crop species,

34 e.g., food crops such as potato (Solanum tuberosum), vegetables such as tomato (Solanum

35 lycopersicum), eggplant (Solanum melongena L.), and pepper (Capsicum annuum), raw industrial

36 materials such as tobacco (Nicotiana tabacum) [1, 2], and certain plant models used in research (e.g.,

37 Nicotiana spp., Solanum spp., Petunia spp., and Datura spp.) [3, 4]. Therefore, Solanaceae plants play

38 an important role in agricultural economics and scientific research [5-8].

39 The eggplant, exclusively native to the Old World, belongs to the largest genus of the

40 Solanaceae, Solanum, and has been listed by the Food and Agriculture Organization as the fourth

41 largest vegetable crop. The world production of eggplants was approximately 52.3 million tons in

42 2017, with China being the main producer. Previous studies of the eggplant focused on the evolution

43 [9-12], genetic linkage map [13, 14], molecular marker development [15, 16], resistance [17, 18], fruit

44 quality [19, 20], and high-throughput genotyping [20, 21]. 
However, given the lack of comprehensive studies on the eggplant genome, only 775 pathogen recognition genes have been reported in the eggplant, compared to more than 1,000 genes in each of the three other Solanaceae crops (tomato, pepper, and potato) [22], which influences the progress of studies on the evolution of disease resistance in different Solanaceae plants [23]. Eggplants are the richest source of chlorogenic acid (CGA; 5-O-caffeoylquinic acid) [24, 25]. This dietary phenolic acid has been proven to exhibit anti-inflammatory, antimutagenic, and antiproliferative activities; however, the mechanism of CGA formation in the eggplant has not been well elucidated [26, 27]. Therefore, a high-quality reference genome is urgently needed for eggplant research. Two published eggplant references (SME_r2.5.1 and Eggplant_V3) [13, 28] were obtained by mainly employing the Illumina short-read sequencing technology, thus exhibiting assembly fragmentation and significant gap sizes.

To facilitate our understanding of the eggplant biology and evolution, we generated a chromosome-scale reference genome assembly of a cultivated eggplant variety, 'guiqie1', and analyzed the sequence in comparison with those of other members of the Solanaceae. Our work provides the fundamental information for unraveling the evolution and domestication of the eggplant and may ultimately lead to further improvement of this important worldwide crop.

\section{Results and Discussion}

\section{Genome sequencing, assembly, and annotation}

63 We performed genome sequencing of the eggplant with the PacBio Sequel platform using a set of 15

64 SMRTcells, which yielded a total of $114.5 \mathrm{~Gb}$ of data (average polymerase read length: $14.5 \mathrm{~kb}$ )

65 (Table S1). The PacBio-only assembly contained 625 contigs, with a total length of $1,155.8 \mathrm{Mb}$ and

66 an $\mathrm{N} 50$ length of $5.3 \mathrm{Mb}$ (maximum contig length: $21.7 \mathrm{Mb}$ ) (Table 1). Subsequently, we used

67 Dovetail Hi-C data $(80.7 \mathrm{~Gb})$ to refine this assembly. Of the 625 contigs, 318 were sorted into 12

68 superscaffolds, accounting for $97.1 \%$ of the original 1,155.8-Mb assembly. The superscaffolds were

69 further anchored to 12 linkage groups to form pseudochromosomes (Figure S1), with N50 of $93.9 \mathrm{Mb}$

70 and a maximum length of $112 \mathrm{Mb}$ (Table 1$)$. The number of pseudochromosomes $(n=12)$

71 corresponded to the number of chromosomes in the eggplant and many members of the Solanaceae

$72[29,30]$.

74 Table 1 Comparison of eggplant assemblies.

\begin{tabular}{llll}
\hline Assembly feature & $\begin{array}{l}\text { New assembly } \\
\text { (guiqie1) }\end{array}$ & Eggplant_V3 & SME_r2.5.1 \\
\hline
\end{tabular}




\begin{tabular}{llll}
\hline Size of assembly & $1,155.8 \mathrm{Mb}$ & $1,474,9 \mathrm{Mb}$ & $833.1 \mathrm{Mb}$ \\
Number of scaffolds & 319 & 10,383 & 33,873 \\
Contig N50 & $5.3 \mathrm{Mb}$ & $16.7 \mathrm{~kb}$ & $14.3 \mathrm{~kb}$ \\
Pseudochromosome/scaffold N50 & $93.9 \mathrm{Mb}$ & $100.4 \mathrm{Mb}$ & $64.5 \mathrm{~kb}$ \\
Longest & $112 \mathrm{Mb}$ & $142 \mathrm{Mb}$ & $630 \mathrm{~kb}$ \\
pseudochromosome/scaffold & & & 35.7 \\
GC content (\%) & 36.1 & 36.0 & 70.4 \\
Repeat content (\%) & 70.1 & 73 & 85,446 \\
Number of genes & 35,018 & 34,916 & $39.6 \mathrm{Mb}(4.75 \%)$ \\
Size of Ns/gaps (\%) & $32.5 \mathrm{~kb}(0.003 \%)$ & $416.4 \mathrm{Mb}(28.23 \%)$ &
\end{tabular}

75 Eggplant_V3 assembly was downloaded from

76 https://solgenomics.net/organism/Solanum_melongena/genome

78 Benchmarking Universal Single-Copy Ortholog (BUSCO) evaluations of the genome sequence

79 revealed $96.2 \%$ completeness. Compared with the previously published eggplant genomes

80 (SME_r2.5.1 and Eggplant_V3) [13, 28], which both mainly employed the Illumina short-read

81 sequencing technology, resulting in more fragmented assemblies (contig N50 lengths: 14.3 and 16.7

$82 \mathrm{~kb}$, respectively) and larger gap sizes (Ns: $4.75 \%$ and $28.23 \%$, respectively), our genome assembly

83 achieved a great improvement in both quality and integrity (Table 1 and Table S2).

84 To validate the superscaffolds, we mapped the 952 DNA markers of linkage map LWA2010 [31]

85 to the eggplant assembly with BWA-MEM [32] and obtained the best mapped position for each

86 marker; a total of 946 (99.4\%) markers could be mapped onto the 12 superscaffolds (Table S3). Then,

87 ALLMAPS [33] was used with default parameters to assign the superscaffolds to each

88 pseudochromosome, and a high value of the Pearson correlation coefficient ( $\rho$-value $>0.9$ ) between

89 the physical position and map location of genetic markers indicated a high quality of the eggplant

90 assembly (Figure S2). We also aligned the markers of linkage map LWA2010 to the Eggplant_V3

91 assembly and found that $832(87.4 \%)$ markers could be assigned to the 12 pseudochromosomes

92 (Table S4), which was less than that obtained using our data (99.4\%). Generally, the

93 pseudochromosomes showed a good collinearity between the new eggplant and Eggplant_V3

94 assemblies (Figure 1 and Table S5). 


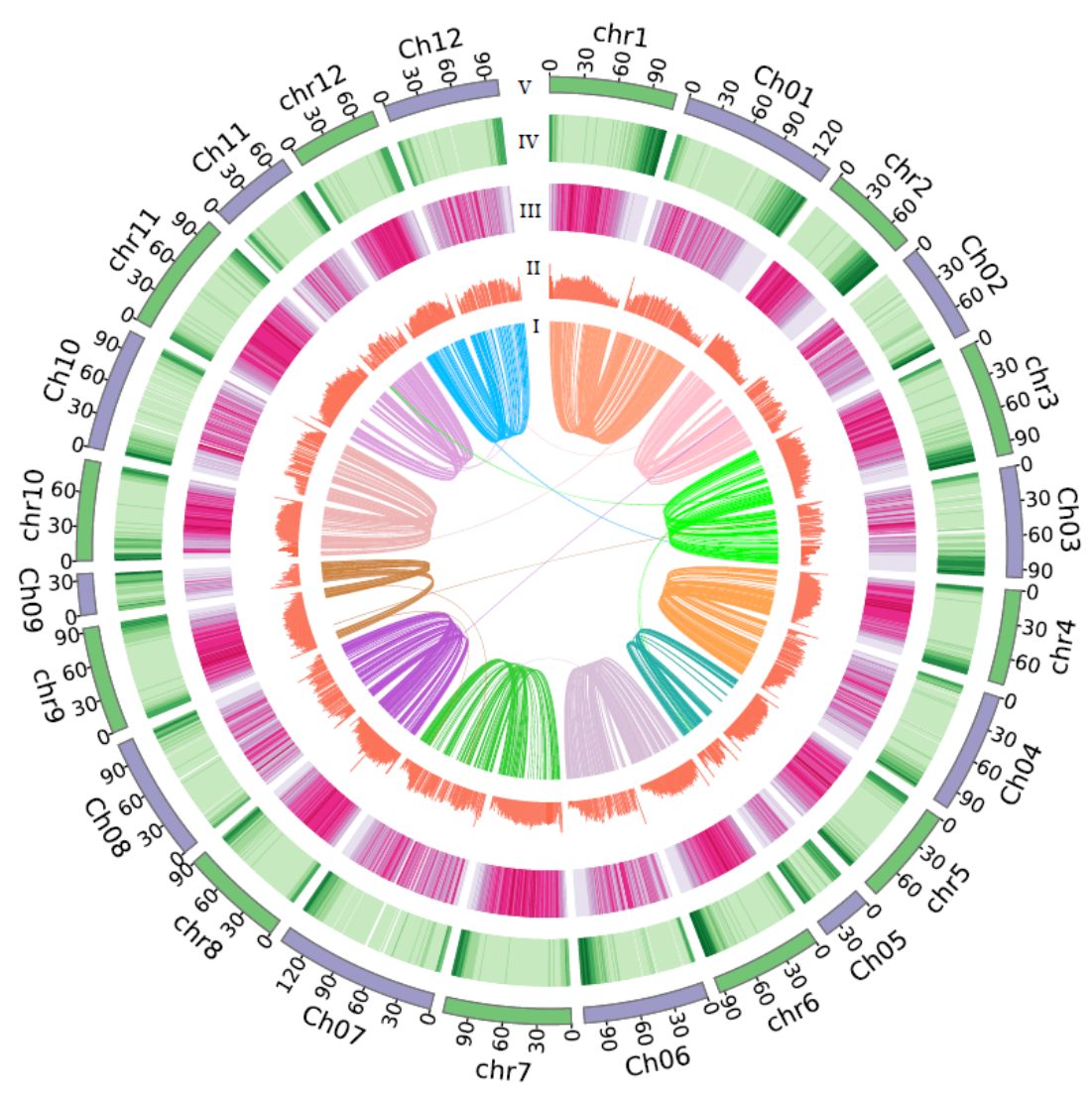

97 Figure 1 Comparison of the eggplant assemblies. I: Syntenic alignments between the new eggplant

98 assembly and Eggplant_V3 assembly based on one-to-one orthologous genes processed by MCscan

99 (Python version) with a C-score cutoff of 0.99 (links). II: GC content in non-overlapping 1-Mb

100 windows (histograms). III: Percent coverage of transposable elements in non-overlapping 1-Mb

101 windows (heat maps). IV: Gene density calculated on the basis of the number of genes in

102 non-overlapping 1-Mb windows (heat maps). $\mathrm{V}$ : Lengths of pseudochromosomes $(\mathrm{Mb})$ of the new eggplant assembly (green) and Eggplant_V3 assembly (purple).

A total of $70.1 \%$ of the assembly was annotated as repetitive sequences using a combination of homology-based and de novo approaches (Table S6). This proportion was consistent with that reported previously [28]. Transposable elements (TEs) play an important role in shaping eukaryotic genomes and driving their evolution [34]. In the eggplant, TEs accounted for $68.9 \%$ of the genome size, with long terminal repeats (LTRs) being the most predominant type (63.9\% of the genome size) (Table S7). The proportions of TEs and LTRs were both less than those in the pepper [29, 35] and more than those in tomato [30] and potato [36]. The most abundant LTRs were the Gypsy elements 
112 (52\%), followed by Copia (7.9\%) (Table S7). This scenario was also observed in the sequenced

113 pepper genome, indicating that the LTRs/Gypsy elements were the major driving force for the

114 expansion of the eggplant genome. We then examined the insertion time of all LTRs based on

115 sequence divergence. The eggplant appeared to have undergone a surge of retrotransposon

116 amplification approximately 0.124 million years ago (Figure S3), suggesting that the expansion event

117 was quite recent during its genome evolution.

118

119

120

121

122

123

124

125

126

127

128

129

130

131

132

133

134

135

136

137

138

To facilitate genome annotation of eggplant genes, we sequenced RNA samples from roots, stems, leaves, and flowers. The sequencing data were imported to the gene prediction pipeline, which also integrated homology-based and de novo strategies. We predicted 35,018 protein-coding genes, with an average gene length of 5,068 bp and an average of 4.7 exons per gene (Table S8). This number of genes is almost the same as that in tomato (35,768 genes), potato (39,028 genes), and pepper $(35,845)$, indicating similar numbers of genes in this clade. The distribution of gene density was inversely correlated with TEs (Figure 1). BUSCO assessment of the predicted gene sets suggested $96.6 \%$ completeness, of which $94.2 \%$ and $2.4 \%$ were single-copy and duplicated genes, respectively (Table S9), suggesting the integrity of our new eggplant gene annotation. Further functional annotation using public databases indicated that 31,963 (91.3\%) genes could be classified using at least one of the databases and 19,466 (55.6\%) genes could be annotated using all five databases (Table S10). In addition, a total of 6,520 noncoding RNAs (ncRNAs) were found in the eggplant genome, including 116 microRNAs (miRNAs), 1,254 transfer RNAs (tRNAs), 4,629 ribosomal RNAs (rRNAs), and 521 small nuclear RNAs (snRNAs) (Table S11).

\section{Genome comparison and gene family evolution}

Genome collinearity analysis of Solanaceae plants showed that some chromosomes were conserved; in particular, chromosomes 2, 6, and 7 retained a large percentage of collinear regions among eggplant, pepper, potato, and tomato (Figures 2a, S4). 
bioRxiv preprint doi: https://doi.org/10.1101/824540; this version posted October 31, 2019. The copyright holder for this preprint (which was not certified by peer review) is the author/funder, who has granted bioRxiv a license to display the preprint in perpetuity. It is made available under aCC-BY-NC-ND 4.0 International license.
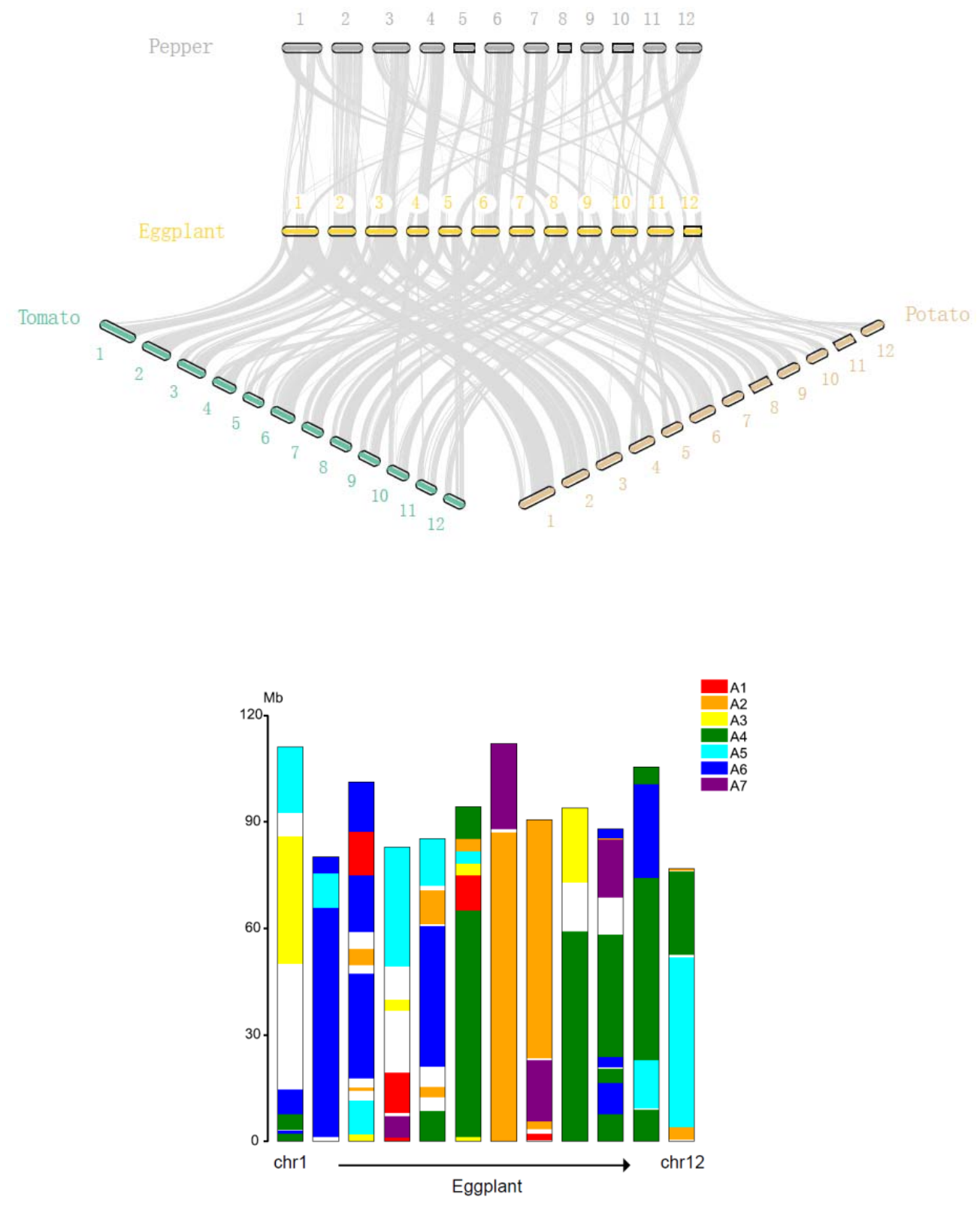
bioRxiv preprint doi: https://doi.org/10.1101/824540; this version posted October 31, 2019. The copyright holder for this preprint (which was not certified by peer review) is the author/funder, who has granted bioRxiv a license to display the preprint in perpetuity. It is made available under aCC-BY-NC-ND 4.0 International license.

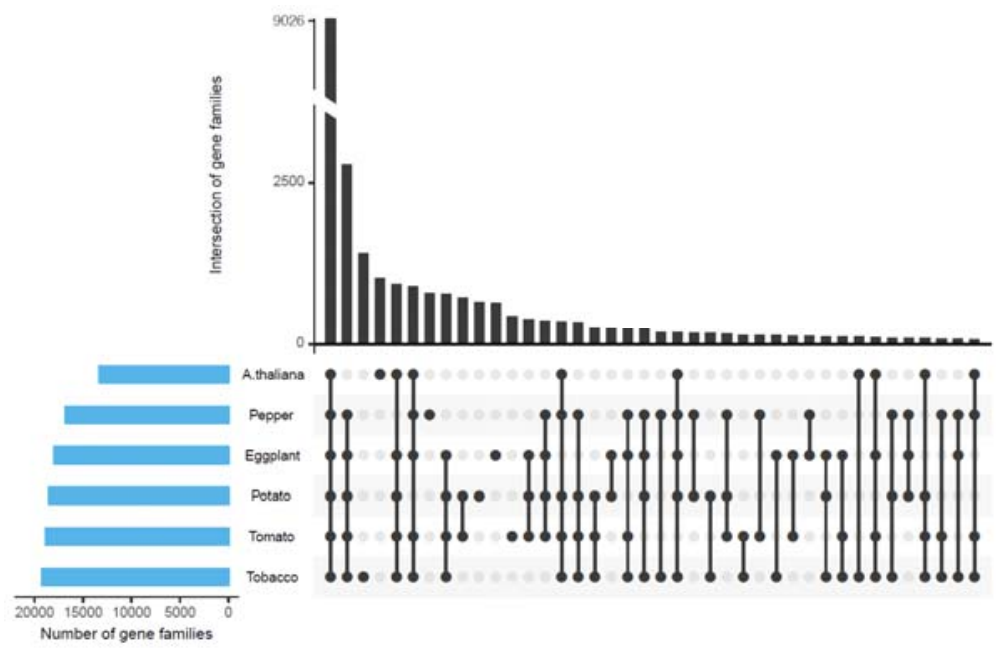

145

(d)

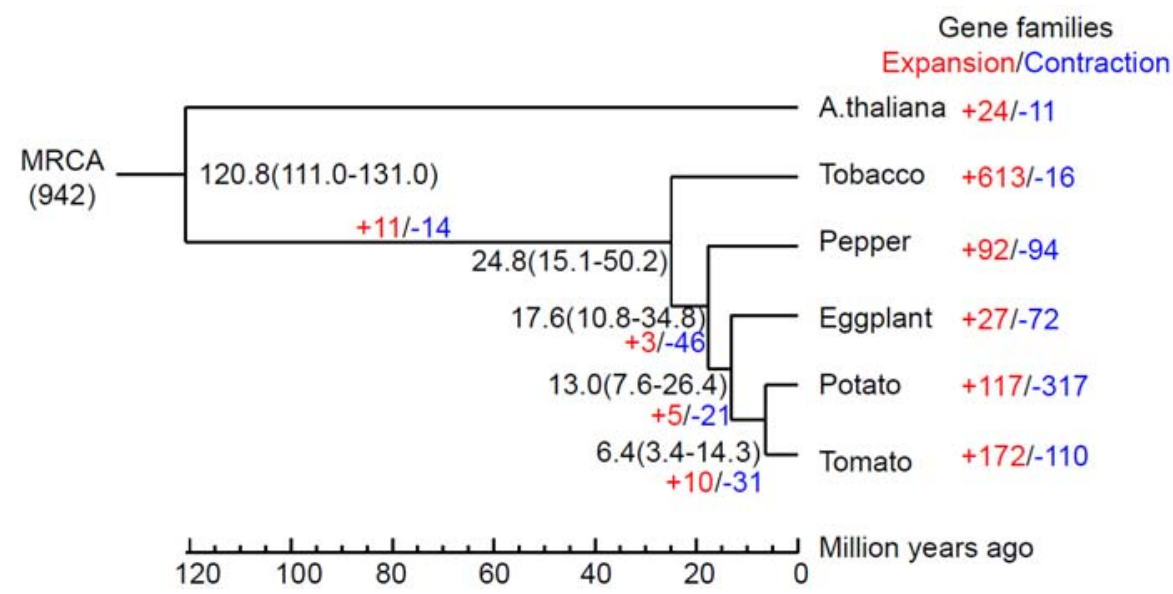

147

(e) 


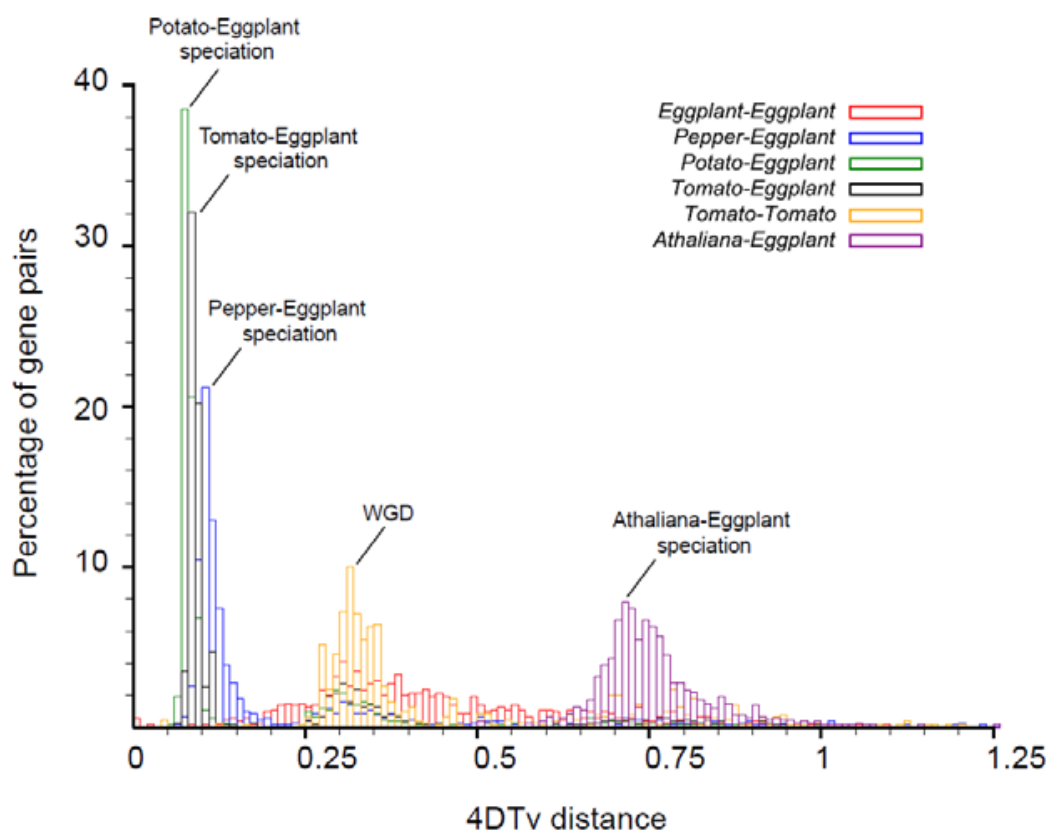

149 Figure 2 Comparative analysis and evolution of the eggplant genome. (a) Analysis of the synteny

150 among Solanaceae genomes. Macrosynteny connecting blocks of $>30$ one-to-one gene pairs is shown.

151 (b) Genome evolution of the eggplant from the ancestral eudicot karyotype (AEKPre- $\gamma$ ) of seven

152 protochromosomes. Colors indicate the origin from the seven AEKPre- $\gamma$ protochromosomes. White

153 spaces represent chromosomal regions where ancestral origin was not assigned. (c) Intersections of

154 gene families between six plant species (eggplant, pepper, potato, tobacco, tomato, and Arabidopsis

155 thaliana). The figure was plotted using UpSetR [40], with the rows representing gene families and the

156 columns representing their intersections. For each set that is part of a given intersection, a black filled

157 circle is placed in the corresponding matrix cell. If a set is not part of the intersection, a light gray

158 circle is shown. A vertical black line connects the topmost black circle with the bottommost black

159 circle in each column to emphasize the column-based relationships. The size of the intersection is

160 shown as a bar chart placed on top of the matrix so that each column lines up with exactly one bar. A

161 second bar chart, showing the size of each set, is shown to the left of the matrix. (d) Phylogenetic tree

162 with divergence times and history of orthologous gene families. Numbers on the nodes represent

163 divergence times, with the error range shown in parentheses. The numbers of gene families that

164 expanded (red) or contracted (blue) in each lineage after speciation are shown on the corresponding

165 branch. MRCA, most recent common ancestor. (e) Genome duplication in Solanaceae genomes

166 (pepper, tomato, potato, and eggplant) revealed by 4DTv analysis.

Based on the ancestral and lineage-specific whole-genome duplications reported for eudicots [37], we inferred genome evolution of the eggplant and other Solanaceae plants from the ancestral 
170

171

172

173

174

175

176

177

178

179

180

181

182

183

184

185

186

187

188

189

190

191

192

193

194

195

196

197

198

199

200

201

202

203

eudicot karyotype (AEKPre- $\gamma$ ) of seven protochromosomes. Figure $2 b$ shows the chromosomes of the eggplant, with the seven protochromosomes of AEKPre- $\gamma$ depicted in different colors. The map of the chromosomal regions that originated from different ancestral eudicot karyotypes (AEKs) is similar among eggplant, potato, and tomato (Figure S5 and Table S12) but much different from that of pepper. The pepper genome contains more predicted chromosomal regions, indicating that the genome of the pepper has undergone a much different process of genomic rearrangements to reach its current structure of 12 chromosomes, compared with that of the genomes of the other three solanaceous species.

We clustered the protein-coding genes of eggplant, pepper, potato, tobacco, tomato, and Arabidopsis thaliana into gene families (Table S13) and identified 25,620 gene families, of which 9,026 were shared by all six species. The intersections of the gene families are illustrated in Figure $2 \mathrm{c}$. There are 358 gene families shared among the eggplant, pepper, potato, and tomato. In the eggplant, 26,596 genes were clustered into 17,926 gene families, of which 646 families were species-specific. Annotation of these specific genes showed various functions (Tables S14, S15), but they were particularly overrepresented in the chitin-related Gene Ontology (GO) categories. Chitin-binding genes are known as a pathogenesis-related gene family, which plays a fundamental role in the defense response of plants $[38,39]$. This finding suggests possible response roles, related to biotic stress, in eggplant.

Analysis of evolution of gene families revealed that 27 gene families were expanded and 72 gene families were contracted in the eggplant (Figure 2d and Tables S16-S19). For the six plants, 799 single-copy genes were used to construct a phylogenetic tree and estimate their divergence times (Figure 2d). The data showed that the eggplant was separated from potato and tomato $\sim 12$ million years ago during the Solanaceae evolution.

We then deduced whole-genome duplication (WGD) events in the eggplant based on the distribution of the distance-transversion rate at fourfold degenerate sites (4DTv methods) of paralogous gene pairs (Figure 2e). After the eggplant-A. thaliana speciation (peak at $\sim 0.71$ ), there occurred a common Solanaceae WGD event (peak at $~ 0.31$ ). The divergence of eggplant-pepper occurred at a peak of $\sim 0.1$, followed by eggplant-tomato $(4 \mathrm{dTv}=0.08)$ and eggplant-potato $(4 \mathrm{dTv}=$ 0.07) divergence, which is consistent with the phylogenetic analysis. There is no evidence of an eggplant-specific WGD after the differentiation of Solanum plants.

In addition, we used the bidirectional best hit (BBH) method and recovered a total of 8,982 one-to-one orthologous gene sets among the five Solanaceae plants for positive selection gene (PSG) detection. In the eggplant, 364 PSGs were identified $[P<0.05$, likelihood ratio test (LRT)], which were especially enriched in GO terms related to intermembrane lipid transfer (three PSGs), regulation 
204 of transcription, DNA-templated (24 PSGs), and DNA-binding transcription factor (TF) activity (16

205 PSGs) (Tables S20, S21).

206

\section{Identification of genes involved in disease resistance}

208

209

210

211

212

213

214

215

216

217

218

219

220

221

\begin{tabular}{llllllllllllll}
\hline \multirow{3}{*}{ Species } & \multicolumn{3}{l}{ NBS encoding } & & & \multicolumn{1}{c}{ RLP } & RLK & TM-CC & Total \\
& NBS & CNL & TNL & CN & TN & NL & TX & Others & & & & \\
\hline Eggplant & 82 & 65 & 21 & 18 & 12 & 75 & 11 & 1 & 84 & 511 & 143 & 1,023 \\
Tomato & 64 & 66 & 22 & 13 & 9 & 83 & 13 & 1 & 87 & 533 & 148 & 1,039 \\
Potato & 100 & 90 & 35 & 33 & 12 & 148 & 30 & 4 & 156 & 562 & 111 & 1,281 \\
Pepper & 282 & 137 & 19 & 75 & 15 & 238 & 19 & 7 & 203 & 687 & 151 & 1,833
\end{tabular}

NBS, nucleotide-binding site; CC, coiled-coil; LRR, leucine-rich repeat; TIR, Toll/interleukin-1 receptor; TM, 
There were 15 RGAs overlapped with PSGs, including nine RLK-encoding RGAs, three encoding transmembrane coiled-coil-containing proteins, two encoding NBS-LRR-containing proteins, and one encoding a TIR-NBS-LRR-containing protein (Table S23). Among these, eight genes could be assigned to known resistance genes using the reference PR proteins from the latest PRGdb [44]. We inferred that these positively selected resistance genes probably played a fundamental role in eggplant self-defense. Further mining revealed an interesting orthoMCL group (129 genes), whose analysis indicated explosive gene expansion in eggplant (21 genes) and pepper (96 genes), in contrast to tomato (three genes) and potato (two genes). Tobacco had seven members in this group, while Arabidopsis did not have any. All of these genes were annotated using PRGdb as encoding bacterial spot resistance gene BS2 (Table S24) [45]. In a maximum-likelihood phylogenetic tree, constructed using IQ-TREE [46], the 21 eggplant genes formed a monophyletic cluster (Figure S3) and, moreover, were found to be tandemly clustered at the head of chromosome 12 . We inferred that the occurrence of these genes might be a consequence of tandem duplication events during eggplant genome evolution, which was also observed in pepper [29].

\section{Identification of genes involved in CGA synthesis}

243 CGAs (esters of certain trans-cinnamic acids and quinic acid) are major phenolic metabolites in the eggplant, which typically account for $80 \%$ to $95 \%$ of total hydroxycinnamic acids in the fruit flesh $[47,48]$. CGAs play a role in plant defense and as antioxidants and are accumulated in many Solanaceae plants $[47,49]$. However, the CGA content in the eggplant has been reported to be roughly 10 and 100 times higher than that in tomato and potato, respectively [50]. CGA is well known to be beneficial for human health, mainly owing to its antioxidant, anti-inflammatory, antipyretic, anticarcinogenic, antimicrobial, analgesic, neuroprotective, cardioprotective, hypotensive, anti-obesity, and antidiabetic properties [48, 51]. Moreover, CGA is highly stable at high temperatures, and its content increases after cooking [52]. Thus, eggplant is considered to be the best source of CGA among the Solanaceae.

The biosynthesis of CGA occurs in eggplants through the phenylpropanoid pathway, which involves six key enzymes $[47,53]$. The three initial steps, catalyzed by phenylalanine ammonia-lyase $(P A L)$, cinnamate 4-hydroxylase $(C 4 H)$, and 4-coumaroyl-CoA ligase $(4 C L)$, produce the intermediate p-coumaroyl-CoA (Figure 3a). Using homologous gene comparison, we identified six $P A L$, two $C 4 H$, and five $4 C L$ candidate genes in the eggplant genome (Figures 3b, S7 and Table S25). Arabidopsis contains four PAL genes, two of which (AtPAL1 and AtPAL2) are associated with lignin and flavonoid

259 biosynthesis [54]. Three eggplant $P A L$ genes were in three distinct phylogenetic groups, and the other 260 three clustered together, while the four Arabidopsis PAL genes formed a single clade (Figure S8). 261 Overexpression of AtPAL2 in tobacco resulted in a twofold increase in the CGA content [55]. C4H is 
262 a cytochrome P450 (CYP) monooxygenase from the CYP73A subfamily, and only one member,

263 designated CYP73A5, exists in Arabidopsis. One $C 4 H$ gene (EGP13151) in eggplant exhibited more

264 sequence identity with the Arabidopsis gene than did the other (EGP24021) (86\% versus 65\%,

265 respectively). Missense mutations in $C 4 H$ result in metabolic changes, threatening plant survival [54,

266 56]. Downregulation of $C 4 H$ resulted in a decrease of CGA levels in tobacco, as well as in a feedback

267 inhibition of $P A L$ activity [57]. It has been reported that Arabidopsis contains four $4 C L$ genes, two of

268 which are involved in lignin biosynthesis, one is related to flavonoid biosynthesis, and the last one

269 preferentially towards erulate and sinapate instead of 4-coumarate [54]. The eggplant has five 4CL

270 genes, which is similar to the number in the other three Solanaceae members but is only half of that in

271 tobacco (Figure 3b). Phylogenetic analysis revealed that each 4CL was in a distinct clade (Figure S9).

272 A previous study has shown that the expression levels of $P A L, C 4 H$, and $4 C L$ in eggplants at the

273 commercially ripe stage were notably higher in the fruit flesh and skin than in other tissues, indicating

274 their correlation with the higher CGA content in the fruit [50].

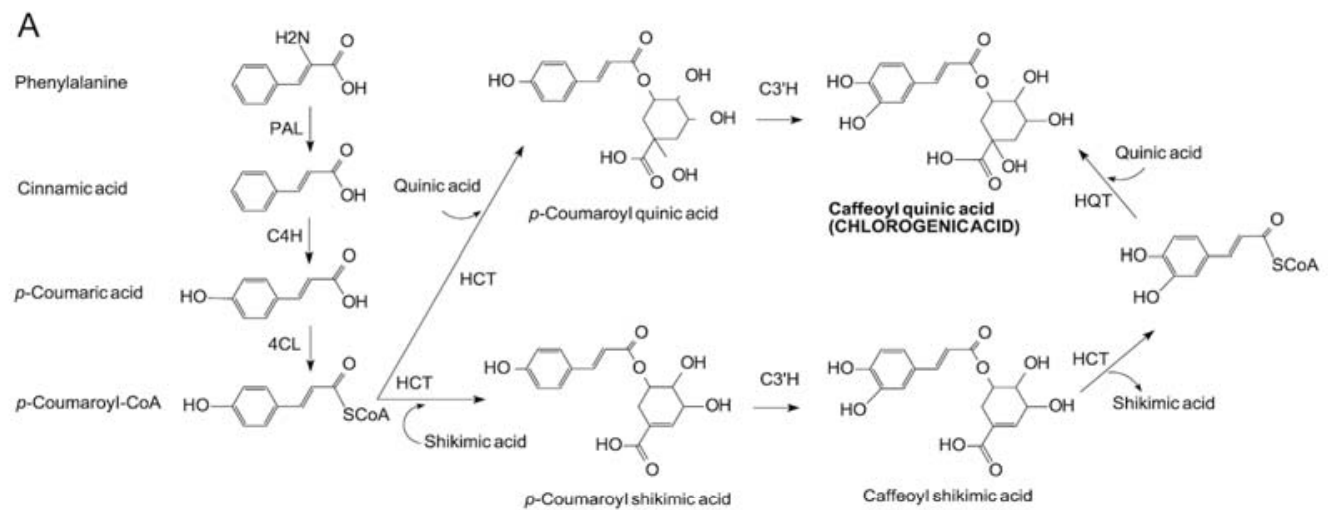

B

277 Figure 3 Genes involved in chlorogenic acid (CGA) synthesis. (a) Biochemical pathway for CGA

278 synthesis in the eggplant. The enzymes involved are as follows: $P A L$, phenylalanine ammonia-lyase;

$279 C 4 H$, cinnamate 4-hydroxylase; 4CL, 4-coumaroyl-CoA ligase; $H C T$,

280 hydroxycinnamoyl-CoA:shikimate hydroxycinnamoyl transferase; $C 3^{\prime} H$, $p$-coumaroyl ester 
281

282

283

284

285

3'-hydroxylase; $H Q T$, hydroxycinnamoyl-CoA:quinate hydroxycinnamoyl transferase. (b)

Orthologous genes involved in CGA biosynthesis from eggplant (red), tomato (green), potato (purple), pepper (light green), tobacco (blue), and Arabidopsis (yellow), identified using orthoMCL, followed by manual inspection. Each circle represents one gene.

After the three initial steps in CGA biosynthesis, two possible pathways have been suggested (Figure 3a): (1) $p$-coumaroyl-CoA is converted into $p$-coumaroyl quinic acid with quinic acid via hydroxycinnamoyl-CoA:shikimate hydroxycinnamoyl transferase $(H C T)$, followed by hydroxylation to form CGA via $p$-coumaroyl ester $3^{\prime}$-hydroxylase $\left(C 3^{\prime} H\right)$; and (2) $p$-coumaroyl-CoA is converted into $p$-coumaroyl shikimic acid with shikimic acid via $H C T$, followed by hydroxylation to form caffeoyl shikimic acid via $C 3^{\prime} H$. Caffeoyl shikimic acid, catalyzed by $H C T$, is converted into caffeoyl-CoA, which is then converted into CGA by trans-esterification with quinic acid via hydroxycinnamoyl-CoA:quinate hydroxycinnamoyl transferase (HQT) [58]. HCT and $H Q T$ are closely related BAHD-like acyltransferases $[59,60]$, and both are encoded by single-copy genes in eggplant, tomato, and potato (Figure $3 \mathrm{~b}$ and Table S25). However, $H Q T$ is absent in Arabidopsis and pepper. Overexpression of $H Q T$ in AtPAL2-overexpressing tobacco plants resulted in a 1.4-fold increase in the CGA content, while silencing of $H Q T$ resulted in a $~ 50 \%$ reduction in CGA [55]. In tomato, overexpression of $H Q T$ led to an increase in CGA accumulation, improving the plant antioxidant capacity and bacterial pathogen resistance [61]. RNAi suppression of $H Q T$ in potato resulted in a $\sim 90 \%$ reduction in CGA and early flowering [62]. In the eggplant, the expression of $H Q T$ was the strongest in the fruit flesh and skin, compared with that in other tissues at the ripe stage [50]. $C 3^{\prime} H$ is a CYP monooxygenase belonging to the CYP98A subfamily; in Arabidopsis [63], $\mathrm{C3}^{\prime} \mathrm{H}$ (designated CYP98A3) is one of three members of this family (the other two members are AT1G74540-CYP98A8 and AT1G74550-CYP98A9). Unlike Arabidopsis, multiple homologs of $C 3^{\prime} H$ were detected in the five Solanaceae species, including five $C 3^{\prime} H$ genes in the eggplant. Similar to $4 C L$, each $C 3^{\prime} H$ was located in a distinct phylogenetic clade (Figure S10). We inferred that these gene duplications had evolved via independent processes, which led to divergent gene functions or neofunctionalization, responsible for the remarkable increase of CGA biosynthesis in the eggplant.

Polyphenol oxidases (PPOs), which oxidize specific phenolic substrates released from vacuoles upon tissue damage to highly reactive quinones, play key roles in plant defense mechanisms against pests and pathogens $[64,65]$. However, oxidation of these high-level phenolics, including CGA, results in flesh browning, which negatively affects the apparent quality of eggplants [48]. In this respect, simultaneous breeding for a high CGA content and low PPO activity would result in cultivars with better fruit quality and reduced flesh browning [47]. We identified nine PPOs in the eggplant, with eight genes tandemly clustered at the end of chromosome 8 and one located on chromosome 2 (Table S26 and Figure S7). Previously published studies discovered six PPO genes in the eggplant 
317 [65], and five, except PPO6, could be anchored to chromosome 8 using a linkage map [48]. Protein

318 sequence identities ranged from $92 \%$ to $99 \%$ when comparing these six genes to our dataset (Table

319 S27). We further examined PPOs in other species. There were nine, eight, eight, and twelve PPO

320 homologs in tomato, potato, pepper, and tobacco, respectively (Figure S11). The absence of PPOs in

321 Arabidopsis has been discussed [66]. We also observed that the distribution patterns of PPO genes in

322 the tomato and potato genomes were highly similar to that in the eggplant genome, with one located

323 on chromosome 2 and the rest clustered at the end of chromosome 8 (Table S26), indicating a highly

324 conserved synteny among the three solanaceous species.

\section{Identification of genes encoding transcription factors}

Plant secondary metabolism is regulated by TFs, which act as transcriptional activators or repressors $[67,68]$. We identified 1,702 TF-encoding genes in the eggplant, representing $4.86 \%$ of the total genes. The number of members from each TF family in the eggplant was comparable to that in four other plants but was much lower than that of certain families in tobacco, such as bHLH, ERF, and NAC (Table S28). Genes encoding MYB TFs, containing conserved MYB DNA-binding domains, are a large family of functionally diverse genes, which can be classified into four subfamilies, 1R, R2R3, 3R, and 4R [67]. The R2R3 subfamily is the largest and considered to comprise the major phenylalanine-derived compound modulators in plants. We identified $121 \mathrm{MYB}$ and 61 MYB-related TFs in the eggplant, of which 112 belonged to the R2R3 subfamily, and most of them could be categorized into 20 subgroups (Table S29) according to the previously characterized R2R3 genes in Arabidopsis [69, 70]. Several subgroups (SG4-SG7) have been found to regulate the phenylpropanoid pathway, including anthocyanin and flavonol biosynthesis [67]. We identified three SG3, four SG4, three SG6, and three SG7 genes in the eggplant. The SmMyb1 gene, belonging to SG6, was reported to regulate CGA accumulation and anthocyanin biosynthesis [50]. No SG5 members were identified based on the current criteria. We also found a gene cluster, which was located at the end of chromosome 7 and contained five members, four belonging to SG2 and one belonging to SG3, suggesting their key roles in regulating self-defense [71, 72].

\section{Conclusion}

346 We sequenced and assembled the genome of the eggplant and greatly improved the quality and 347 integrity of the sequence compared with those of previously published draft sequences. As a vital crop 348 in the Solanaceae, eggplants are cultivated and consumed worldwide. However, there have been much 349 fewer studies of the eggplant than of other members of the Solanaceae, such as tomato and potato, 350 which have been established as biological models for studying the development of fleshy fruits and 
351 tubers, respectively. The main reason is due to the lack of a high-quality reference genome of the

352

353

354

355

356

357

358

359

360

361

362 eggplant. Although a genome sequence of the inbred eggplant line ' $67 / 3$ ' has been published recently, our assembly showed several advantages, including a longer contig N50 (5.3 Mb vs. $16.7 \mathrm{~kb}$ ), fewer total scaffolds ( 319 vs. 10,383$)$, and a much smaller size of gaps $(0.003 \%$ vs. $28.23 \%)$. Genome validation using a linkage map confirmed a high accuracy of our assembly.

We comprehensively characterized genes involved in disease resistance, CGA synthesis, and polyphenol oxidation, as well as those encoding TFs, thus demonstrating a significant value of the reference genome sequence. We also conducted comparative analysis of the eggplant genome with those of four other species of the Solanaceae and Arabidopsis. This study will facilitate the breeding of eggplant cultivars with strong disease resistance, high nutritional value, and low browning.

\section{Methods}

\section{Sample preparation}

Guiqie1 (S. melongena) plants were collected from the Vegetable Research Institute, Guangxi Academy of Agricultural Science ( $28^{\circ} \mathrm{N}$ and $118^{\circ} \mathrm{E}$ ), Guangxi province, China. Roots, stems, leaves, and flowers of Guiqie1 were harvested, immediately frozen in liquid nitrogen, and stored at $-80{ }^{\circ} \mathrm{C}$ until use. Genomic DNA was isolated from leaf tissues using the DNeasy plant mini kit (Qiagen). RNA was extracted using the RNeasy plant mini kit (Qiagen).

\section{DNA sequencing}

Illumina short-read sequencing

Purified DNA was sheared using a focused ultrasonicator (Covaris) and then used for 350-bp paired-end library construction with the Next Ultra DNA library prep kit (NEB) for Illumina sequencing. Sequencing was performed on the Illumina NovaSeq platform.

SMRT long-read sequencing

SMRTbell DNA libraries ( 20 kb) were prepared using the BluePippin size selection system following the officially released PacBio protocol. Long reads were generated using the PacBio Sequel system.

Hi-C library construction and sequencing 
380 A Hi-C library was prepared using the Dovetail Hi-C library preparation kit. Briefly, nuclear

381

382

383

384

385 chromatin was fixed in young eggplant seedlings with formaldehyde and extracted. Fixed chromatin was digested with DpnII, and sticky ends were filled in with biotinylated nucleotides and ligated. Then, crosslinks were reversed, and purified DNA was treated to remove any free biotin from ligated fragments. DNA was then sheared to a size of $\sim 350 \mathrm{bp}$, and biotinylated fragments were enriched through streptavidin bead pulldown, followed by PCR amplification to generate the library. The library was sequenced on the Illumina NovaSeq platform.

\section{Genome assembly and evaluation}

A diploid contig assembly of the eggplant genome was carried out using FALCON, followed by FALCON-Unzip, integrated in the pb-assembly tool suite (v0.0.4). The resulting assembly contained primary contigs (partially phased haploid representation of the genome) and haplotigs (phased alternative alleles for a subset of the genome). Two rounds of contig polishing were performed. For the first round, as part of the FALCON-Unzip pipeline, primary contigs and secondary haplotigs were polished using haplotype-phased reads and the Quiver consensus caller. For the second round of polishing, we concatenated the primary contigs and haplotigs into a single reference and then mapped all raw reads to the combined assembly reference using pbmm2 (v0.12.0), followed by consensus calling with Arrow (GenomicConsensus v2.3.3). After a draft set of contigs was generated, the Dovetail Hi-C kit was run for Hi-C-based scaffolding with cloud-based HiRise software [73]. Finally, Pilon (v1.22) was used to correct errors introduced into the assembly from long reads.

To assess the completeness of the assembled eggplant genome, we performed BUSCO analysis by searching against the conserved 1,440 Embryophyta gene set (v3.0, lineage dataset embryophyta_odb9).

\section{Repeat annotation}

Tandem repetitive sequences were identified within the eggplant genome using Tandem Repeats Finder (v4.07). The interspersed repeats were determined using a combination of homology-based and de novo approaches. The homology-based approach, with the RepBase (v21), was used to identify TEs by searching against the eggplant genome assembly at the DNA and protein levels using RepeatMasker (v4.0.7; http://www.repeatmasker.org/) and ProteinRepeatMask (v4.0.7), respectively. A de novo repeat library was customized using RepeatModeler (v1.0.8) and LTR_FINDER (v1.0.6) [74] and then imported to RepeatMasker to identify repetitive elements. Additionally, the results from LTR_FINDER were integrated, and false positives were removed from the initial predictions using the LTR_retriever 
413 pipeline [75]. The insertion time was estimated as $\mathrm{T}=\mathrm{K} / 2 \mu$, where $\mathrm{K}$ is the divergence rate, and $\mu$ is the

414 neutral mutation rate. A neutral substitution rate of $9.6 \times 10^{-9}$ was used for the eggplant [76].

\section{Gene annotation}

417 Protein-coding gene predictions were conducted through a combination of homology-based, de novo, 418 and transcriptome-based prediction methods. Proteins for six plant genomes (A. thaliana, C. annuum, 419 S. tuberosum, $N$. tabacum, S. lycopersicum, and S. melongena SME_r2.5.1) were downloaded from

420 Phytozome (release 13), the National Center for Biotechnology Information (NCBI), and the Eggplant

421 Genome DataBase. Protein sequences were aligned to the assembly using genblasta (v1.0.4).

422 GeneWise (v2.4.1) was used to predict the exact gene structure of the corresponding genomic regions 423 on each genblasta hit. Three ab initio gene prediction programs, Augustus (v3.2.1), GlimmerHMM

424 (v3.0.4), and SNAP (v2006-07-28), were used to predict coding regions in the repeat-masked genome.

425 Finally, RNA-seq data were mapped to the assembly using hisat2 (v2.0.1); stringtie (v1.2.2) and

426 TransDecoder (v3.0.1) were then used to assemble the transcripts and identify candidate coding

427 regions in gene models. All gene models predicted by the above three approaches were combined

428 using EvidenceModeler into a non-redundant set of gene structures. The produced gene models were

429 finally refined using PASA v2.3.3. Functional annotation of protein-coding genes was achieved using

430 BLASTP (E-value: 1e-05) against two integrated protein sequence databases, SwissProt and

431 TrEMBL. Protein domains were annotated using InterProScan (v5.30). The GO terms for each gene

432 were extracted with InterProScan. The pathways in which genes might be involved were assigned

433 using BLAST against the KEGG database (release 84.0), with an E-value cutoff of 1e-05.

434 Four types of ncRNAs, namely, miRNAs, tRNAs, rRNAs, and snRNAs, were annotated. The

435 tRNA genes were predicted using tRNAscan-SE (v1.3.1). The rRNA fragments were predicted

436 through alignment to Arabidopsis and rice template rRNA sequences using BlastN (v2.2.24), with an

437 E-value of 1e-5. The miRNA and snRNA genes were determined by searching against the Rfam

438 database (release 12.0) using INFERNAL (v1.1.1).

\section{Genome comparison and gene family and phylogenetic analyses}

441 The AEK genes in the modern genome of the grape were obtained from Murat et al. [37]. Based on

442 genome alignments using the cumulative identity percentage and cumulative alignment length

443 percentage BLAST parameters [77], we identified homologous genes of AEK in the modern genomes

444 of Solanaceae plants. Synteny blocks between the genomes of Solanaceae plants were detected using

445 the GRIMM-Synteny software (http://grimm.ucsd.edu/GRIMM/), with groups of fewer than five 
446 genes filtered out; then, the synteny blocks were assigned to the seven protochromosomes based on the homologous genes of AEK.

OrthoMCL (v2.0.9) [78] was used to cluster gene families from A. thaliana, C. annuum, $S$. tuberosum, N. tabacum, S. lycopersicum, and S. melongena. CAFÉ (v3.1) [79] was used to determine

450 gene family expansion and contraction.

451 A total of 799 single-copy genes were used to construct a phylogenetic tree for the six plant 452 genomes. Fourfold degenerate sites were extracted from each family and concatenated to form one 453 supergene for each species. The GTR-gamma substitution model was selected, and PhyML (v3.0) 454 [80] was used to reconstruct the phylogenetic tree. The divergence times among the six plants were 455 estimated using the MCMCtree program (v4.4) as implemented in the Phylogenetic Analysis of 456 Maximum Likelihood (PAML) package, with an independent rate clock and the JC69 nucleotide 457 substitution model. The calibration times of divergence between A. thaliana and S. lycopersicum 458 (111-131 million years ago) were obtained from the Time Tree database [81].

To detect PSGs in the eggplant genome, one-to-one orthologs were identified among the six plants using BLASTP, based on the BBH method with a sequence coverage $>30 \%$ and identity $>30 \%$, followed by selection of the best match. A total of 8,982 one-to-one orthologous gene sets were found among C. annuum, S. tuberosum, N. tabacum, S. lycopersicum, and S. melongena. The branch-site model incorporated in the PAML package was used, with the eggplant used as the foreground branch and pepper, potato, and tomato used as background branches. The null model used in the branch-site test assumed that the $\mathrm{Ka} / \mathrm{Ks}$ values for all codons in all branches were $\leq 1$, whereas the alternative model assumed that the foreground branch included codons evolving at $\mathrm{Ka} / \mathrm{Ks}>1$. A maximum LRT was used to compare the two models. The $P$-value was calculated using the chi-squared distribution with one degree of freedom, and then $P$-values were adjusted for multiple testing using the false discovery rate (FDR) method. Genes were identified as positively selected when FDR was $<0.05$.

470 Furthermore, we required that at least one amino acid site possessed a high probability of being positive selected (Bayes probability $>95 \%$ ). If no amino acid in PSG passed this cutoff, such gene was identified as false positive and excluded. GO enrichment was derived using Fisher's exact test and 473 adjusted using the Benjamini-Hochberg method with the cutoff set at $P<0.05$.

\section{Identification of disease resistance genes}

476 The RGAugury pipeline (https://bitbucket.org/yaanlpc/rgaugury) [43] was used to screen the entire 477 gene set for RGA prediction. The default $P$-value cutoff for initial RGA filtering was set to le-5 for 478 BLASTP. 


\section{Identification of CGA synthesis-related genes and phylogenetic analysis}

481 To identify CGA synthesis-related genes, homologous Arabidopsis genes were mined from the

482 literature and downloaded. Corresponding gene family results were extracted and manually inspected.

483 HMMER or BLASTP were used whenever necessary. Protein sequences were aligned using muscle

484 (v3.8.31). Maximum-likelihood phylogenetic trees were constructed using IQ-TREE (v1.6.11), with

4851,000 bootstrap replicates, and further illustrated in MEGA (v7.0.26).

486

487 Identification and classification of TFs

488 The Plant Transcription Factor Database v5.0 (planttfdb.cbi.pku.edu.cn) was used to identify TFs

489 [82]. R2R3-MYB TFs were further characterized using the corresponding members in Arabidopsis

490 [67, 69], and motifs were verified using MEME (v5.0.5) [83]. Subgroups were designated as

491 previously reported $[67,70]$.

492

493 Availability

494 The genome assembly and the sequencing data used for de novo whole-genome assembly are

495 available from the China National GeneBank (CNGB) Nucleotide Sequence Archive (CNSA) under

496 accession number CNP0000734.

497

$498 \quad$ Conflict of interest

499 The authors declare no conflict of interest.

500

501 References

502 1. Mueller, L.A., et al., The SOL Genomics Network: a comparative resource for Solanaceae biology and

503 beyond. Plant Physiol, 2005. 138(3): p. 1310-7.

$5042 . \quad$ Liu, J., et al., Erratum to: Improving the resistance of eggplant (Solanum melongena) to Verticillium

505 wilt using wild species Solanum linnaeanum. Euphytica, 2015. 206(3): p. 825-826. 
506 3. Doganlar, S., et al., Conservation of gene function in the solanaceae as revealed by comparative

507 mapping of domestication traits in eggplant. Genetics, 2002. 161(4): p. 1713.

508 4. Knapp, S., M.S. Vorontsova, and J. Prohens, Wild Relatives of the Eggplant (Solanum melongena L.:

509 Solanaceae): New Understanding of Species Names in a Complex Group. Plos One, 2013. 8(2).

$5105 . \quad$ Rinaldi, R., et al., New Insights on Eggplant/Tomato/Pepper Synteny and Identification of Eggplant and

511 Pepper Orthologous QTL. Frontiers in Plant Science, 2016. 7(2016).

512 6. Saski, C., et al., Complete chloroplast genome sequences of Hordeum vulgare, Sorghum bicolor and

513 Agrostis stolonifera, and comparative analyses with other grass genomes. Theoretical and Applied

$514 \quad$ Genetics, 2007. 115(4): p. 571-590.

515

7. Walker, P.J., Understanding genomic evolution and segregation distortion in Solanaceae: A COSII

$516 \quad$ linkage map in. Dissertations \& Theses - Gradworks, 2009.

517

8. Daniell, H., et al., Complete chloroplast genome sequences of Solanum bulbocastanum, Solanum lycopersicum and comparative analyses with other Solanaceae genomes. Theoretical \& Applied Genetics, 2006. 112(8): p. 1503.

520

9. Acquadro, A., L. Barchi, and P. Gramazio, Coding SNPs analysis highlights genetic relationships and evolution pattern in eggplant complexes. Plos One, 2017. 12(7): p. e0180774.

10. Page, A., et al., Eggplant domestication: pervasive gene flow, feralisation and transcriptomic divergence. Mol Biol Evol, 2019.

11. Weese, T.L. and L. Bohs, Eggplant origins: Out of Africa, into the Orient. Taxon, 2010. 59(1): p. 49-56.

12. Wei, Q., et al., Comparative Transcriptome Analysis in Eggplant Reveals Selection Trends during Eggplant Domestication. Int J Genomics, 2019. 2019: p. 7924383.

13. Barchi, L., et al., A chromosome-anchored eggplant genome sequence reveals key events in Solanaceae evolution. Sci Rep, 2019. 9(1): p. 11769.

14. Hirakawa, H., et al., Draft Genome Sequence of Eggplant (Solanum melongena L.): the Representative Solanum Species Indigenous to the Old World. DNA Research, 2014. 21(6): p. 649-660. 
534 16. Gramazio, P., et al., Transcriptome analysis and molecular marker discovery inSolanum incanumandS.

535

536

537

538

539

540

541

542

543

544 aethiopicum, two close relatives of the common eggplant (Solanum melongena) with interest for breeding. Bmc Genomics, 2016. 17(1): p. 300.

17. Zhou, X.H., et al., De Novo Sequencing and Analysis of the Transcriptome of the Wild Eggplant Species Solanum Aculeatissimum in Response to Verticillium dahliae. Plant Molecular Biology Reporter, 2016. 34(6): p. 1193-1203.

18. Zhou, X., et al., Molecular Cloning and Characterization of a Wild Eggplant Solanum aculeatissimum NBS-LRR Gene, Involved in Plant Resistance to Meloidogyne incognita. Int J Mol Sci, 2018. 19(2).

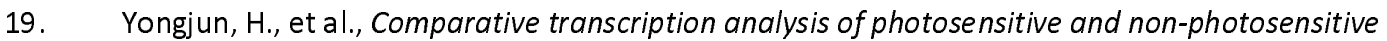
eggplants to identify genes involved in dark regulated anthocyanin synthesis. BMC genomics, 2019. 20(1).

20. San José, R., et al., Composition of eggplant cultivars of the Occidental type and implications for the improvement of nutritional and functional quality. International Journal of Food Science \& Technology, 2013. 48(12): p. 2490-2499.

21. Lorenzo, B., et al., Single Primer Enrichment Technology (SPET) for High-Throughput Genotyping in Tomato and Eggplant Germplasm. Frontiers in plant science, 2019. 10.

22. Di Donato, A., et al., Investigation of orthologous pathogen recognition gene-rich regions in solanaceous species. Genome, 2017. 60(10): p. 850-859.

23. Andolfo, G., et al., Defining the full tomato $N B-L R R$ resistance gene repertoire using genomic and CDNA RenSeq. BMC Plant Biology, 2014. 14(1): p. 120.

24. Whitaker, B.D. and J.R. Stommel, Distribution of hydroxycinnamic acid conjugates in fruit of commercial eggplant (Solanum melongena L.) cultivars. Journal of Agricultural and Food Chemistry, 2003. 51(11): p. 3448-3454.

25. Alarcon-Flores, M.I., et al., Systematic Study of the Content of Phytochemicals in Fresh and Fresh-Cut Vegetables. Antioxidants (Basel), 2015. 4(2): p. 345-58.

26. Youn, Y., et al., Chlorogenic acid-rich Solanum melongena extract has protective potential against rotenone-induced neurotoxicity in PC-12 cells. J Food Biochem, 2019: p. e12999.

27. Meinhart, A.D., et al., Study of new sources of six chlorogenic acids and caffeic acid. Journal of Food Composition and Analysis, 2019. 82: p. 13.

28. Hirakawa, H., et al., Draft genome sequence of eggplant (Solanum melongena L.): the representative solanum species indigenous to the old world. DNA Res, 2014. 21(6): p. 649-60. 
$56529 . \quad$ Kim, S., et al., Genome sequence of the hot pepper provides insights into the evolution of pungency in Capsicum species. Nat Genet, 2014. 46(3): p. 270-8.

567 30. Tomato Genome, C., The tomato genome sequence provides insights into fleshy fruit evolution.

$568 \quad$ Nature, 2012. 485(7400):p. 635-41.

31. Fukuoka, H., et al., Development of gene-based markers and construction of an integrated linkage map in eggplant by using Solanum orthologous (SOL) gene sets. Theor Appl Genet, 2012. 125(1): p.

32. Li, H., Aligning sequence reads, clone sequences and assembly contigs with BWA-MEM. arXiv:1303.3997v2 [q-bio.GN], 2013.

33. Tang, H., et al., ALLMAPS: robust scaffold ordering based on multiple maps. Genome Biol, 2015. 16: $\mathrm{p}$. 3.

34. Feschotte, C., N. Jiang, and S.R. Wessler, Plant transposable elements: where genetics meets genomics. Nat Rev Genet, 2002. 3(5): p. 329-41.

35. Qin, C., et al., Whole-genome sequencing of cultivated and wild peppers provides insights into Capsicum domestication and specialization. Proc Natl Acad Sci U S A, 2014. 111(14): p. 5135-40.

36. Potato Genome Sequencing, C., et al., Genome sequence and analysis of the tuber crop potato. Nature, 2011. 475(7355): p. 189-95.

37. Murat, F., et al., Reconstructing the genome of the most recent common ancestor of flowering plants. Nat Genet, 2017. 49(4): p. 490-496. Pepper (Capsicum annuum L.) and Transcriptional Regulation to Phytophthora capsici, Abiotic Stresses and Hormonal Applications. Int J Mol Sci, 2018. 19(8). sets and their properties. Bioinformatics, 2017. 33(18): p. 2938-2940. 
595 43. Li, P., et al., RGAugury: a pipeline for genome-wide prediction of resistance gene analogs (RGAs) in plants. BMC Genomics, 2016. 17(1): p. 852.

44. Osuna-Cruz, C.M., et al., PRGdb 3.0: a comprehensive platform for prediction and analysis of plant disease resistance genes. Nucleic Acids Res, 2018. 46(D1): p. D1197-D1201.

45. Tai, T.H., et al., Expression of the Bs2 pepper gene confers resistance to bacterial spot disease in tomato. Proc Natl Acad Sci U S A, 1999. 96(24): p. 14153-8.

46. Nguyen, L.T., et al., IQ-TREE: a fast and effective stochastic algorithm for estimating maximum-likelihood phylogenies. Mol Biol Evol, 2015. 32(1): p. 268-74.

47. Plazas, M., et al., Breeding for chlorogenic acid content in eggplant: interest and prospects. Notulae Botanicae Horti Agrobotanici Cluj-Napoca, 2013. 41(1): p. 26-35.

48. Gramazio, P., et al., Location of chlorogenic acid biosynthesis pathway and polyphenol oxidase genes in a new interspecific anchored linkage map of eggplant. BMC Plant Biol, 2014. 14: p. 350.

49. Korkina, L., Phenylpropanoids as naturally occurring antioxidants: from plant defense to human health. Cellular \& Molecular Biology, 2007. 53(1): p. 15-25. Genes and Regulation by a MYB Transcription Factor. Front Plant Sci, 2015. 6: p. 1233. natural polyphenol chlorogenic acid. Biol Pharm Bull, 2006. 29(11): p. 2236-40. content and the inhibitory effect on human neutrophil burst. J Agric Food Chem, 2010. 58(6): p.

53. Vogt, T., Phenylpropanoid biosynthesis. Mol Plant, 2010. 3(1): p. 2-20.

54. Fraser, C.M. and C. Chapple, The phenylpropanoid pathway in Arabidopsis. Arabidopsis Book, 2011. 9: p. e0152.

55. Chang, J., J. Luo, and G. He, Regulation of polyphenols accumulation by combined overexpression/silencing key enzymes of pheny/propanoid pathway. Acta Biochim Biophys Sin (Shanghai), 2009. 41(2): p. 123-30. 
624 57. Blount, J.W., et al., Altering expression of cinnamic acid 4-hydroxylase in transgenic plants provides

58. Ferro, A.M., et al., Impact of novel SNPs identified in Cynara cardunculus genes on functionality of proteins regulating phenylpropanoid pathway and their association with biological activities. BMC

59. D'Auria, J.C., Acyltransferases in plants: a good time to be BAHD. Curr Opin Plant Biol, 2006. 9(3): p. 331-40.

60. Lallemand, L.A., et al., A structural basis for the biosynthesis of the major chlorogenic acids found in coffee. Plant Physiol, 2012. 160(1): p. 249-60.

61. Niggeweg, R., A.J. Michael, and C. Martin, Engineering plants with increased levels of the antioxidant

62. Payyavula, R.S., et al., Synthesis and regulation of chlorogenic acid in potato: Rerouting

63. Schoch, G., et al., CYP98A3 from Arabidopsis thaliana is a 3'-hydroxylase of phenolic esters, a missing

64. Docimo, T., et al., Insights in the Fruit Flesh Browning Mechanisms in Solanum melongena Genetic

65. Shetty, S.M., A. Chandrashekar, and Y.P. Venkatesh, Eggplant polyphenol oxidase multigene family: Lines with Opposite Postcut Behavior. J Agric Food Chem, 2016. 64(22): p. 4675-85.

66. Tran, L.T., J.S. Taylor, and C.P. Constabel, The polyphenol oxidase gene family in land plants:

67. Dubos, C., et al., MYB transcription factors in Arabidopsis. Trends Plant Sci, 2010. 15(10): p. 573-81.

68. Zhou, H., et al., Activator-type R2R3-MYB genes induce a repressor-type R2R3-MYB gene to balance anthocyanin and proanthocyanidin accumulation. New Phytol, 2019. 221(4): p. 1919-1934. Opin Plant Biol, 2001. 4(5): p. 447-56. from Arabidopsis thaliana. Plant J, 1998. 16(2): p. 263-76. 
654 71. Ding, Z., et al., Transgenic expression of MYB15 confers enhanced sensitivity to abscisic acid and

655 improved drought tolerance in Arabidopsis thaliana. J Genet Genomics, 2009. 36(1): p. 17-29.

656

72. Agarwal, M., et al., $A R 2 R 3$ type $M Y B$ transcription factor is involved in the cold regulation of CBF genes and in acquired freezing tolerance. J Biol Chem, 2006. 281(49): p. 37636-45.

658

73. Putnam, N.H., et al., Chromosome-scale shotgun assembly using an in vitro method for long-range linkage. Genome Res, 2016. 26(3): p. 342-50.

74. Xu, Z. and H. Wang, LTR_FINDER: an efficient tool for the prediction of full-length LTR retrotransposons. Nucleic Acids Res, 2007. 35(Web Server issue): p. W265-8.

75. Ou, S. and N. Jiang, LTR_retriever: A Highly Accurate and Sensitive Program for Identification of Long Terminal Repeat Retrotransposons. Plant Physiol, 2018. 176(2): p. 1410-1422.

76. Wang, Y., et al., Sequencing and comparative analysis of a conserved syntenic segment in the Solanaceae. Genetics, 2008. 180(1): p. 391-408.

77. Salse, J., et al., Improved criteria and comparative genomics tool provide new insights into grass paleogenomics. Brief Bioinform, 2009. 10(6): p. 619-30.

78. Li, L., C.J. Stoeckert, Jr., and D.S. Roos, OrthoMCL: identification of ortholog groups for eukaryotic genomes. Genome Res, 2003. 13(9): p. 2178-89.

79. De Bie, T., et al., CAFE: a computational tool for the study of gene family evolution. Bioinformatics,

80. Guindon, S., et al., New algorithms and methods to estimate maximum-likelihood phylogenies: assessing the performance of PhyML 3.0. Syst Biol, 2010. 59(3): p. 307-21. organisms. Bioinformatics, 2006. 22(23): p. 2971-2. in plants. Nucleic Acids Res, 2017. 45(D1): p. D1040-D1045.

\section{Supporting information}

681 Additional supporting information may be found online in the Supporting Information section at the 682 end of the article. 